\title{
RFID Technology, Systems, and Applications
}

Radio Frequency Identification (RFID) has been around for more than half a century. It is only in recent years that this technology has begun to attract a lot of attention, due to the convergence of lower cost and increased capabilities of RFID tags. Currently, RFID is emerging as an important technology for revolutionizing a wide range of applications including supply chain management, retail, anti-counterfeiting, baggage handling, and healthcare. Many organizations are planning embrace or have already embraced RFID in their main operations to take advantage of the potential of more automation, efficient business processes, and inventory visibility. While RFID provides promising benefits such as business process automation, significant challenges on information systems and technologies need to be overcome before these benefits can be realized.

This special issue presents the latest developments, trends, and research solutions for RFID technology, which received an enthusiastic response. There were 79 submissions, including three extended from the best papers of IWRT 2009 (the 3rd International Workshop on RFID Technology, Milan, Italy). Twenty papers were selected after several rounds of review by the guest editors and the invited reviewers. These papers cover a wide range of topics in RFID research that reflect some key directions in this active yet diverse research area.

Handling large volume of RFID data is a challenging task in developing RFID applications. The paper by Yao et al. focuses on complex RFID event processing and the proposed complex event processing framework has been applied in managing surgical operations in an RFID-enabled hospital. The paper by $\mathrm{Ma}$ et al. proposes a grid-based load balancing mechanism for RFID middleware applications. The mechanism solves the overloading issues of RFID ap- plications using two modules, namely buffer management and load balancing management.

Security and privacy are serious concerns in RFID applications. The paper by Gandino et al. focuses on the reader-to-reader collision problem by proposing a Probabilistic Distributed Color System (PDCS) reader-to-reader anti-collision protocol. The proposed protocol allows multichannel transmissions and achieves more efficiency. The paper by PerisLopez et al. gives a thorough analysis on recently proposed RFID authentication protocols and offers some valuable guidelines that need to be followed when designing secure protocols. The paper by Rizzo et al. describes a new electronic sealing system for commercial containers. The system is based on RFID technology and can significantly increase the security of supply chains. The paper by Pietro and Molva proposes an information confinement technique to keep the server compromise limited. An efficient identification technique based on a probabilistic mechanism is also discussed in the paper that enables a server to identify a tag by performing only bitwise operations. The paper by Bueno-Delgado and Vales-Alonso analyzes the identification performance of real RFID systems, considering not only the capture effect, but also the requirements imposed by the EPCglobal Class-1 Gen-2. Finally, the paper by Hancke describes a nearfield, bit-exchange channel design that minimizes latency and allows for more secure distance-bounding measurements in RFID systems.

Deploying RFID systems presents many challenges such as optimization and interference problems. The paper by Chen et al. describes an optimization model for position planning of RFID readers based on a novel multi-swarm particle swarm optimizer. The algorithm proposed in this paper has some potential for solving optimization problems in complex RFID 
networks. The paper by Papapostolou and Chaouchi studies the RFID-assisted indoor localization, particularly focusing on the interference problem. Different interference problems are modeled and a theoretical analysis is conducted to study their impacts on the RFID positioning performance. The paper by $\mathrm{Ku}$ et al. identifies the optimal deployment patterns that guarantee $k$-covering (i.e., at least $k$ RFID tags are accessible anywhere in the deployment region). The paper by Manzanares-Lopez et al. reports the design and implementation of EPCglobal's Object Name Service (ONS) and Discovery Service (DS) based on a distributed architecture, which eliminates the weakness of centralized implementation of ONS and DS.

The paper by Yang et al. describes a hybrid RFID sensor network for efficient resource information management in humanitarian logistics centers. The paper by Worapot et al. reports the work on simulating RFID enabled supply chains, which makes possible for companies to study new business processes without high investment. The paper by Nam and Yeom describes a business-aware framework (BizAF), which offers a middleware to simplify the development of RFID applications based on the EPCglobal network. The paper by Amaral et al. describes an adaptive framework for developing RFID applications on mobile devices. The paper by Najera et al. reports some experience and lessons learned in developing two RFID-enabled healthcare applications.

Finally, the paper by Vo et al. discusses a framework called RADIO-MAMA that uses RFID technology for real-time management of mobile assets. The main feature of this framework is the separation of business logic from sensor technologies for data collection, which offers flexibility in system maintenance. The paper by Chaudhry et al. reviews RFID middleware implementations and proposes a Middleware as a Service (MaaS) architecture that encapsulates underlying complexities of RFID systems from enterprise applications. The paper by Ranasinghe et al. surveys the existing architectural approaches in various stages during a product's life cycle.

We thank all authors for considering this special issue as an outlet to publish their research results in the area of RFID. We would like to thank the referees who provided very useful and thoughtful feedback to the authors. This special issue would not have been possible without their help and selfless dedication. We also would like to thank the Editor in Chief of JNCA, Prof. Mohammed Atiquzzaman, for his continuous support during the review and publication process.

Finally, we hope that this collection of papers can help shed some light and generate fruitful discussions on current and future RFID research and developments.

\section{Guest Editors}

Quan Z. Sheng

School of Computer Science

The University of Adelaide, Australia

Email:qsheng@cs.adelaide.edu.au

Sherali Zeadally

Department of Computer Science and Information Technology

University of the District of Columbia, USA

Email: szeadally@udc.edu

Aikaterini Mitrokotsa

Security and Cryptography Laboraroty (LASEC)

School of Computer and Communication Sciences

EPFL, Switzerland

Email: mitrokatkm@gmail.com

Zakaria Maamar

College of Information Technology

Zayed University, UAE

Email:zakaria.maamar@zu.ac.ae 